\title{
Calcium Oxalate Urolithiasis
}

National Cancer Institute

\section{Source}

National Cancer Institute. Calcium Oxalate Urolithiasis. NCI Thesaurus. Code C123242.

Urolithiasis in which the composition of the stones is predominantly calcium oxalate. 